\title{
Evidence of MAPK-JNK1/2 activation by hepatitis $E$ virus ORF3 protein in cultured hepatoma cells
}

\author{
Mohammad Khalid Parvez $\cdot$ Mohammed \\ Salem Al-Dosari
}

Received: 14 May 2014/Accepted: 3 September 2014/Published online: 4 October 2014

(C) Springer Science+Business Media Dordrecht 2014

\begin{abstract}
Hepatitis E virus (HEV) has recently emerged to cause chronic infection in some immunosuppressed individuals, including extrahepatic manifestations in acute and chronic patients. Mammalian MAPK-JNK1/2 is expressed in hepatocytes, which is known to be involved in anti-apoptotic signaling pathway for the establishment of persistent infection. Though in vitro modulation of cellular MAPK-ERK cascade by HEV-ORF3 protein is suggested to have a role in host pathobiology, activation of the JNK module has not been studied so far. In this report, we have shown for the first time, evidence of MAPK-JNK1/2 activation by HEV-ORF3, using viral replicon as well as expression vector in human hepatoma cells. Phospho-ELISA based relative quantitaion has demonstrated $\sim 54 \%$ and $\sim 66 \%$ phosphorylation of JNK1/2 in replicon-RNA and ORF3-vector DNA transfected cells, respectively. Our finding however, suggests further molecular studies to validate a role of JNK1/2 in HEV pathogenesis.
\end{abstract}

Keywords Hepatitis E virus · HEV - ORF3 · Replicon $\cdot$ MAPK $\cdot$ JNK1/2

M. K. Parvez $(\bowtie) \cdot$ M. S. Al-Dosari

Department of Pharmacognosy, King Saud University College of Pharmacy, PO Box 2457, Riyadh 11451,

Saudi Arabia

e-mail: khalid_parvez@yahoo.com

\section{Introduction}

Hepatitis E virus (HEV) causes acute hepatitis E, in general as well as chronic infection in some cases of immunocompromised individuals (Holla et al. 2013; Parvez 2013a). HEV infection accounts for global mortality rate of $\sim 2 \%$, including fulminant liver failure in $20-30 \%$ of pregnant women (Purcell and Emerson 2008). Moreover, growing evidences have shown an emerging neuropathic manifestation of the virus in acute and chronic patients, worldwide (Kamar et al. 2011; Tse et al. 2012; Sharma et al. 2013; Peng et al. 2014; Parvez 2014). Human HEV has four recognized genotypes 1, 2, 3 and 4, and of these, HEV3 and HEV4 are recently implicated in the autochthonous sporadic cases of hepatitis E (Parvez 2013a). HEV is a small nonenveloped virus with a plus sense, single-stranded RNA genome that comprises of three open reading frames (ORFs 1, 2 and 3). While ORF1 encodes the nonstructural polyprotein, ORF2 codes for the viral capsid. In animal cells, the ORF3 translates into a very small protein (a.a. 114), phosphorylated at a single serine residue (Ser80) (Jameel et al. 1996). The conserved proline-rich (PXXP) motif in the C-terminal part of ORF3 is recognized to bind with $\mathrm{SH} 3$ domains of host cell-signaling protein modules (Korkaya et al. 2001). HEV-ORF3 has been further demonstrated to have some host-regulatory functions (Holla et al. 2013), including mitogen-activated protein kinase (MAPK)-ERK1/2 pathway, in vitro (Korkaya et al. 2001; Kar-Roy et al. 2004) as well as crucial in establishing in vivo infection (Emerson et al. 2006). 
The MAPK-c-Jun amino-terminal kinase (JNK) or stress-activated protein kinase (SAPK) pathway is activated by ultraviolet light, cytokines, osmotic shock, inhibitors of DNA, RNA, and protein synthesis, and to a lesser extent by growth factors. In contrast to activation of ERK, upstream signal transduction mechanisms for the JNK is generally, less understood. Of the three known mammalian JNK genes, JNK1 $(\mathrm{SAPK} \gamma)$ and JNK2 $\left(\mathrm{SAPK}_{\alpha}\right)$ are expressed in hepatocytes that are known to be involved in anti-apoptotic or pro-cell survival signaling pathway for the establishment of persistent infection (Malhi and Gores 2008). Further, mouse hepatitis virus (MHV), the prototype coronavirus $(\mathrm{CoV})$ is reported to have potentiality to chronically infect the central nervous system in rodents (Maeda et al. 1995). In addition, Mizutani et al. (2005) have demonstrated a role of JNK cascade during persistent SARS-CoV infection in cultured cells. Though in vitro interaction of viral ORF3 with ERK1/2 cascade has indicated its possible role in host pathobiology (Korkaya et al. 2001; KarRoy et al. 2004), involvement of the JNK1/2 has not been described so far. To explore this, we therefore looked into modulation of MAPK-JNK phosphoprotein by HEV-ORF3, if any, in cultured hepatoma cells.

\section{Materials and methods}

Cells and plasmid constructs

Human hepatoma cells, S10-3 (kind gift of Dr. Suzanne U Emerson, NIH, Bethesda, MD, USA) were grown in Dulbecco's modified Eagle's medium (Invitrogen, Carlsbad, CA, USA) supplemented with $2 \mathrm{mM}$ L-glutamine and $9 \%$ fetal bovine serum (Invitrogen) in a $\mathrm{CO}_{2}$ incubator at $37{ }^{\circ} \mathrm{C}$ (Emerson et al. 2004). Cultured cells were seeded in 6-well plates $\left(1.0 \times 10^{6}\right.$ cells/well $)$ for transfections or in 96-well plates $\left(0.5 \times 10^{4}\right.$ cells/well $)$ for ELISA. While RNA-transfected cells were maintained in the incubator at $34.5^{\circ} \mathrm{C}$, DNA-transfected cells were continued at $37^{\circ} \mathrm{C}$. The HEV-SAR 5 replicons: pSKE2-WT and $p S K E 2-G A D$ (kind gift of Dr. Suzanne U Emerson, NIH) were used for genomic RNAtransfections (Emerson et al. 2004), and expression vectors: pTriEX-ORF3 and pTriEx-ORF2 (Parvez et al. 2011) were used for DNA-transfections.
In vitro transcription and transfections

pSKE2-WT and pSKE2-GAD were linearized and capped transcripts were synthesized (Fig. 1a) in the presence of cap analog as previously described (Emerson et al. 2004). The viral RNA polymerase mutant, pSKE2-GAD and mock-transfected cells served as negative controls. In brief, the $50 \mu \mathrm{l}$ reaction-mixture contained $10 \mu \mathrm{l}$ of $5 \times$ transcription buffer (Invitrogen), $5 \mu \mathrm{l}$ of $100 \mathrm{mM}$ dithiothreitol (Invitrogen), $2 \mu \mathrm{l}$ of $40 \mathrm{U}$ of RNasin/ml (Invitrogen), $5 \mu \mathrm{l}$ of nucleoside triphosphates ( $5 \mathrm{mM} \mathrm{ATP,} \mathrm{CTP,}$ and UTP and $0.5 \mathrm{mM}$ GTP) (Invitrogen), $5 \mu \mathrm{l}$ of $5 \mathrm{mM} \mathrm{3}$ - $O$-Me- $\mathrm{m}^{7} \mathrm{G}\left(5^{\prime}\right)$ pppG (Ambion-Life Technologies, Grand Island, NY, USA), and $2 \mu 1$ of $100 \mathrm{U}$ of $\mathrm{T} 7$ polymerase (Invitrogen). The mixtures were incubated at $37{ }^{\circ} \mathrm{C}$ for $1.5 \mathrm{~h}$, and the integrity and yield of transcripts were determined by agarose gelelectrophoresis. For RNA transfections in 6-well plates, $20 \mu \mathrm{l}$ of ice-cooled transcription mixture was mixed with $8 \mu \mathrm{l}$ of DMRIE-C (Invitrogen) and $400 \mu \mathrm{l}$ of OptiMEM medium (Gibco-Invitrogen). The transfection-mixture was added to the pre-washed wells and the plate was incubated at $34.5^{\circ} \mathrm{C}$ for $6 \mathrm{~h}$, followed by addition of another $1 \mathrm{ml}$ of OptiMem. A day after, OptiMem was replaced with growth medium and incubated further. pTriEX-ORF2 and pTriExORF3 DNA were transfected into S10-3 cells as described previously (Parvez 2013b). Empty vector (pTriEx-1.1) and mock-transfected cells served as negative controls. On day 2, post-transfection (p.t.) while RNA or DNA receiving cells from a well were re-seeded (in duplicate) in 8-chamber glass slides, the rest of the cultures were re-seeded (in triplicate) in two sets (RNA-I \& RNA-II and DNA-I \& DNA-II) in 96-well plates. The transfected cells were then incubated at 34.5 or $37{ }^{\circ} \mathrm{C}$ for another 7 days, while changing the growth medium every other day.

Immune-staining and fluorescence microscopy

On day 4 p.t., HEV replication was determined by double immune-staining of the 8-chamber slide for ORF2 and ORF3 expressions in RNA-transfected cells or single staining for ORF2 or ORF3 in DNAtransfected cells, essentially as described elsewhere (Emerson et al. 2004). In brief, slides were fixed with acetone and washed in $1 \times$ PBS. ORF3-specific rabbit polyclonal antibody and ORF2-specific chimpanzee 


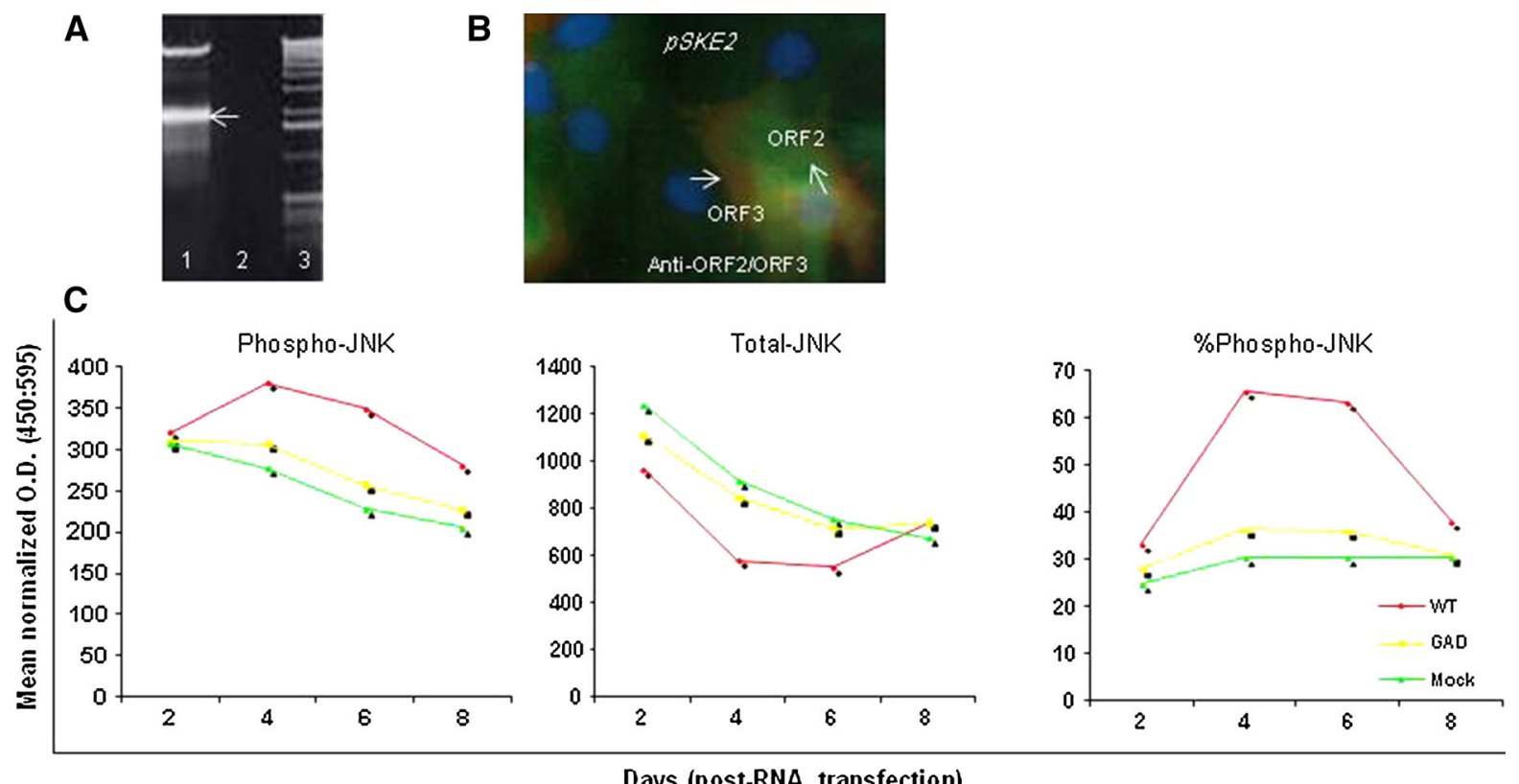

Days (post-RNA transfection)

Fig. 1 Activation of MAPK-JNK by HEV replicon-synthesized ORF3 in S10-3 cells. a Agarose-gel electrophorogram, showing in vitro transcribed viral genomic RNA. Lanes 1 pSKE2; 2 negative control; 3 DNA marker. b Immunefluorescence microscopy (IFM), showing co-expressions of HEV ORF2 [chimp sera $\left(1^{0} \mathrm{Ab} ; 1: 100\right) \&$ Alexa 488-conjugated goat anti-human $\operatorname{IgG}\left(2^{0} \mathrm{Ab} ; 1: 100\right)$ ] and ORF3 [rabbit anti-
ORF3 $\left(1^{0} \mathrm{Ab} ; 1: 500\right)$ \& Alexa 568-conjugated goat anti-rabbit $\left.\left(2^{0} \mathrm{Ab} ; 1: 100\right)\right]$ proteins in RNA-transfected cells at day4. c Phospho-ELISA, showing ex vivo activation (\%) of phosphoJNK1/2 (day4, p.t.) in cells supporting HEV RNA replication and gene expression: wt: pSKE2-wt (red); GAD: pSKE2-GAD (yellow); Mock: untransfeted (green)

instructions. Briefly, the culture medium was removed from each well, and cells were fixed with $100 \mu \mathrm{l}$ of cell fixing buffer (CFB) per well. The plate was sealed and incubated for $20 \mathrm{~min}$ at RT. Cells were washed with washing buffer (WB), and incubated with $100 \mu \mathrm{l}$ of quenching buffer (QB) as above. Cells were washed and further incubated with $100 \mu \mathrm{l}$ of blocking buffer (BB) for $1 \mathrm{~h}$ at RT. After a wash, $50 \mu \mathrm{l}$ of diluted primary phospho-JNK1/2 or total-JNK1/2 specific antibody was added to each well of Set-I and Set-II, respectively. The plate was sealed and incubated overnight at $4{ }^{\circ} \mathrm{C}$. After washing, the cells were incubated with $100 \mu \mathrm{l}$ of diluted secondary antibodies for $1 \mathrm{~h}$ at RT. After a wash with phosphate buffer saline (PBS), $100 \mu$ of pre-warmed developing solution (DS) was added, and the plate was incubate for up to $20 \mathrm{~min}$ at RT in dark until the color developed into medium-to-dark blue. Immediately, $100 \mu \mathrm{l}$ of stop solution (SS) was added per well that changed the color to yellow, and the absorbance was read at $450 \mathrm{~nm}$ (ELex800, BioTek). The colored solution was removed and the plate was washed once with WB and distilled water, and air-dried at RT for 
$5 \mathrm{~min}$. The cells were incubated with $100 \mu \mathrm{l}$ of cell staining buffer (CSB) for $30 \mathrm{~min}$ at RT, followed by washing twice with water. The cells were then incubated with $100 \mu$ of $1 \%$ SDS (w/v, in PBS) at RT for $1 \mathrm{~h}$ on a rocker, and read at $595 \mathrm{~nm}$. Finally, the antibody reading was normalized to the relative cell number by dividing readings of $\mathrm{OD}_{450}$ by $\mathrm{OD}_{595}$, for each well of Set-I and Set-II. The relative quantitation of JNK phosphorylation was determined by normalizing the phospho-JNK1/2 antibody ratio (Set-I; $\mathrm{OD}_{450}: \mathrm{OD}_{595}$ ) to the total-JNK1/2 antibody ratio (Set-II; $\mathrm{OD}_{450}: \mathrm{OD}_{595}$ ) under the same experimental condition. The above experiment was also performed in a time-course manner as well as under serum-free condition.

\section{Results and discussion}

Interactions or/and modulations of host proteins by vector-expressed ORF3 has been widely studied (Panteva et al. 2003; Holla et al. 2013). Of these, a detailed study of regulation of MAPK-ERK cascade in ORF3-expessing stable non-hepatic cells has been reported (Korkaya et al. 2001; Kar-Roy et al. 2004). However, this finding has not been confirmed either in ORF3-expressing hepatocytes or HEV-replicating/infected cells. Moreover, there are no data available so far, on MAPK-JNK modulation by HEV. Therefore, in this report, we, first, utilized the ex vivo genomic-replicon/hepatoma cell system that could mimic the in vivo HEV infection of hepatocytes. In the HEV SAR ${ }_{55}$-replicon system, the RNA replication is manifested on day 4 and peaks on day 6 that declines subsequently (Emerson et al. 2004). However, in the present time-course assay, the optimal JNK phosphorylation was observed on day 4 (p.t.). RNA viruses are known to modulate cellular MAPK pathways, including ERK and JNK cascades, in vivo. Positive immunestaining for ORF2 and ORF3 co-expressions in HEV RNA-transfected cells on day 4, confirmed an active viral replication (Fig. 1b). Further, the ELISA-based relative quantitation of JNK1/2 phosphoprotein in the same culture showed an optimal phosphorylation on day 4 . The JNK1/2 phosphorylation in WT-RNA receiving cells was elevated by about $54 \%$ as compared to GAD-control (Fig. 1c). These experiments, when repeated in serumfree medium under the same conditions did not show significant differences in the results (data not shown).

Although activation of MAP-JNK was clearly observed in HEV-replicating cells, modulatory role of
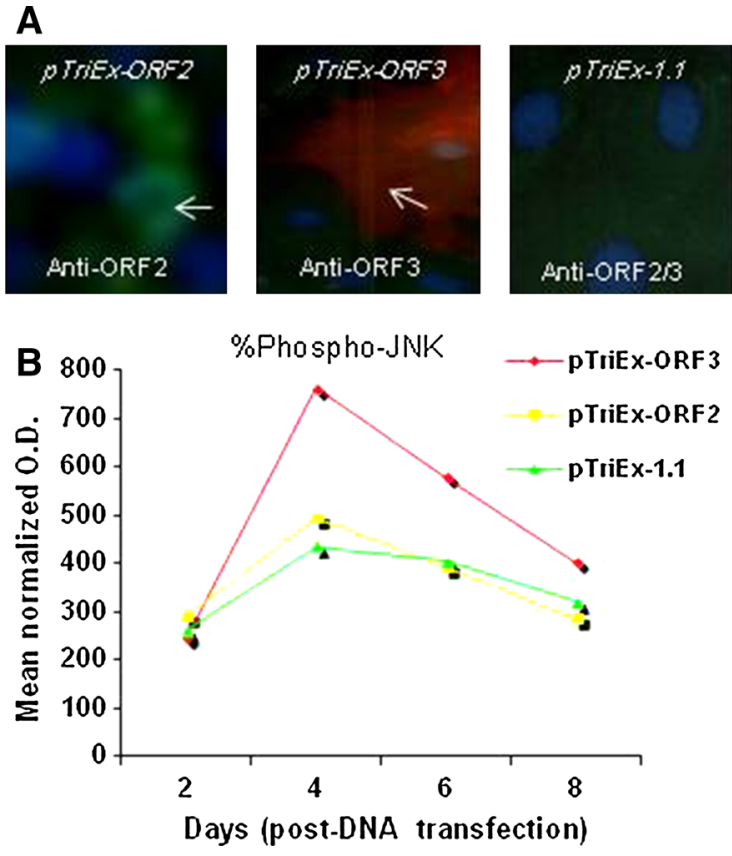

Fig. 2 Activation of MAPK-JNK by over-expressed ORF3 in S10-3 cells. a IFM, showing expressions of HEV ORF2 and ORF3 proteins in vector-transfected cells at day4 p.t. b Ex vivo activation(\%) of phospho-JNK1/2 by ORF3

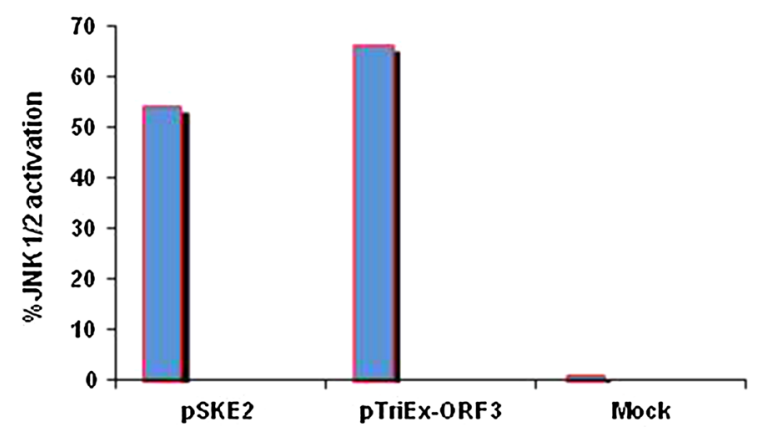

Fig. 3 Comparative modulations of JNK1/2 by viral genomic RNA- and vector-expressed ORF3 in S10-3 cells. pSKE: HEV (genotype 1) genomic replicon RNA; Mock: Untransfected control

ORF3 protein was further investigated in vectortransfected S10-3 cells. Positive immune-staining confirmed the expressions of ORF2 and ORF3 in vectortransfected cells on day 4 (Fig. 2a). A relative quantitation showed activation of JNK1/2 phosphorylation by about $66 \%$ in ORF3 expressing cells, compared to mock-transfected cells (Fig. 2b). There was no evidence of JNK1/2 phosphorylation by ORF2 in $p T r i E x$ - 
ORF2 transfected cells that was comparable to $p T r i E x$ 1.1 receiving cells. However, activation of phosphoJNK $1 / 2$ by vector-expressed ORF3 was approximately $22 \%$ higher than that of viral mRNA-translated ORF3 (Fig. 3). Notably, we had up to $50 \%$ replicon transfection efficiency compared to 18-20\% transfections achieved with expression vectors (data not shown). However, there was much higher production of ORF3 by the customized expression vector as compared to the genomic replicon. Taken together, our data clearly showed regulation of cell-signaling pathway(s) by HEV-ORF3 protein in infected cells that was in conformity with previous reports.

Viruses are known to cause chronic or persistent infections, and regulate cellular MAPK signaling events to evade host-immune responses. Of these, the human immunodeficiency virus (HIV)-Nef, the herpesvirus-TIP, the Epstein-Barr virus (EBV)-latent membrane proteins 1 and 2A (Collette and Olive 1997) were reported previously. Moreover, the hepatitis C virus (HCV)-core (Hayashi et al. 2000; Fukuda et al. 2001) and NS5A proteins (Tan et al. 1999) as well as the HBV-HBx protein has also been shown to activate the MAPK pathway (Benn and Schneider 1994). Further, it has been reported that MAPKJNK1/2 pathway is required for robust replication and/ or persistence of encephalomyocarditis virus (EMCV) (Steer et al. 2006), coxsackievirus B3 (CVB) (Si et al. 2005), MHV (Maeda et al. 1995), SARS-CoV (Mizutani et al. 2005) and enterovirus 71 (EV 71) (Peng et al. 2014). In a recent study, the HBV surface protein (HBsAg) has been demonstrated to selectively interfere the JNK1/2 pathway and provide a mechanism by which HBV can maintain its persistence in infected cells (Wang et al. 2013). In addition to this, involvement of HCV NS3 and NS5A (nonstructural) as well as core and E2 (structural) proteins in JNK pathway is also proposed to mediate pathogenesis of chronic hepatitis C (Rau et al. 2013).

The in vitro interactions of HEV-ORF3 with host proteins that are upstream modulators of PI3K/Akt, PLC $\gamma /$ protein kinase C, and Ras/Raf/MAPK/ERK kinase pathways, have already been demonstrated, previously. In accordance with this, our observation of increased phosphorylation of JNK1/2 in ORF3expressing cells is consistent with the hypothesis that ORF3 might play a crucial role in establishing chronic infection by providing an intracellular pro-survival signal.

\section{Conclusion}

This is the first evidence of modulation of JNK1/2 by HEV-ORF3 protein in infected hepatocytes ex vivo. This may have a possible pro-cell survival role in establishing persistent hepatitis E infection in immunosuppressed individuals or neuro-muscular manifestations of the virus. Nevertheless, further molecular studies are required to validate a role of MAPK-JNK pathway in HEV pathogenic evolution and adaptation.

Acknowledgments The authors would like to extend their sincere appreciation to the Deanship of Scientific Research at King Saud University, Riyadh for its funding to this research through the Research Group Project No. RG-1435-053.

\section{References}

Benn J, Schneider RJ (1994) Hepatitis B virus HBx protein activates Ras-GTP complex formation and establishes a Ras, Raf, MAP kinase signaling cascade. Proc Natl Acad Sci USA 91:10350-10354

Collette Y, Olive D (1997) Non-receptor protein tyrosine kinases as immune targets of viruses. Immunol Today 18:393-400

Emerson SU, Nguyen H, Graff J, Stephany DA, Brockington A, Purcell RH (2004) In vitro replication of hepatitis E virus (HEV) genomes and of an HEV replicon expressing green fluorescent protein. J Virol 78:4838-4846

Emerson SU, Nguyen H, Torian U, Purcell RH (2006) ORF3 protein of hepatitis $\mathrm{E}$ virus is not required for replication, virion assembly, or infection of hepatoma cells in vitro. J Virol 80:10457-10464

Fukuda K, Tsuchihara K, Hijikata M, Nishiguchi S, Kuroki T, Shimotohno K (2001) Hepatitis C virus core protein enhances the activation of the transcription factor, Elk1, in response to mitogenic stimuli. Hepatology 33:159-165

Hayashi J, Aoki H, Kajino K, Moriyama M, Arakawa Y, Hino O (2000) Hepatitis C virus core protein activates the MAPK/ ERK cascade synergistically with tumor promoter TPA, but not with epidermal growth factor or transforming growth factor alpha. Hepatology 32:958-961

Holla RP, Ahmad I, Ahmad Z, Jameel S (2013) Molecular virology of hepatitis E virus. Semin Liver Dis 33:3-14

Jameel S, Zafrullah M, Ozdener MH, Panda SK (1996) Expression in animal cells and characterization of the hepatitis E virus structural proteins. J Virol 70:207-216

Kamar N, Bendall RP, Peron JM, Cintas P, Prudhomme L, Mansuy JM et al (2011) Hepatitis E virus and neurologic disorders. Emerg Infect Dis 17:173-179

Kar-Roy A, Korkaya H, Oberoi R et al (2004) The hepatitis E virus open reading frame 3 protein activates ERK through binding and inhibition of the MAPK phosphatase. J Biol Chem 279:28345-28357

Korkaya H, Jameel S, Gupta D et al (2001) The ORF3 protein of hepatitis E virus binds to Src homology 3 domains and activates MAPK. J Biol Chem 276:42389-42400 
Maeda A, Hayashi M, Ishida K et al (1995) Characterization of DBT cell clones derived from cells persistently infected with the JHM strain of mouse hepatitis virus. J Vet Med Sci 57:813-817

Malhi H, Gores GJ (2008) Cellular and molecular mechanisms of liver injury. Gastroenterology 134:1641-1654

Mizutani T, Fukushi S, Saijo M, Kurane I, Morikawa S (2005) $\mathrm{JNK}$ and PI3k/Akt signaling pathways are required for establishing persistent SARS-CoV infection in Vero E6 cells. Biochim Biophys Acta 1741:4-10

Panteva M, Korkaya H, Jameel S (2003) Hepatitis viruses and the MAPK pathway: is this a survival strategy? Virus Res 92:131-140

Parvez MK (2013a) Chronic hepatitis E infection: risks and controls. Intervirology 56:213-216

Parvez MK (2013b) Molecular characterization of hepatitis E virus ORF1 gene supports a papain-like cysteine protease (PCP)-domain activity. Virus Res 178:553-556

Parvez MK (2014) Hepatitis E virus-induced neuropathy: an emerging extrahepatic manifestation. Future Virol 9:1-4

Parvez MK, Purcell RH, Emerson SU (2011) Hepatitis E virus ORF2 protein over-expressed by baculovirus in hepatoma cells, efficiently encapsidates and transmits the viral RNA to naïve cells. Virol J 8:159

Peng H, Shi M, Zhang L et al (2014) Activation of JNK1/2 and p38 MAPK signaling pathways promotes enterovirus 71 infection in immature dendritic cells. BMC Microbiol 4:147
Purcell RH, Emerson SU (2008) Hepatitis E: an emerging awareness of an old disease. J Hepatol 48:494-503

Rau SJ, Hildt E, Himmelsbach K et al (2013) CD40 inhibits replication of hepatitis $\mathrm{C}$ virus in primary human hepatocytes by c-Jun $\mathrm{N}$ terminal kinase activation independent from the interferon pathway. Hepatology 57:23-36

Sharma B, Nagpal K, Sonnegowda RB, Praksh S (2013) Hepatitis $\mathrm{E}$ with Guillain-Barré syndrome: still a rare association. J Neurovirol 19:186-187

Si X, Luo H, Morgan A et al (2005) Stress-activated protein kinases are involved in coxsackievirus B3 viral progeny release. J Virol 14:13875-13881

Steer SA, Moran JM, Christmann BS et al (2006) Role of MAPK in the regulation of double-stranded RNA- and encephalomyocarditis virus-induced cyclooxygenase-2 expression by macrophages. J Immunol 14:3413-3420

Tan S-L, Nakano H, He Y et al (1999) NS5A, a nonstructural protein of hepatitis $\mathrm{C}$ virus, binds growth factor receptorbound protein 2 adaptor protein in a Src homology 3 domain/ligand-dependent manner and perturbs mitogenic signaling. Proc Natl Acad Sci USA 96:5533-5538

Tse AC, Cheung RT, Ho SL, Chan KH (2012) Guillain-Barré syndrome associated with acute hepatitis E infection. J Clin Neurosci 19:607-608

Wang S, Chen Z, Hu C et al (2013) Hepatitis B virus surface antigen selectively inhibits TLR2 ligand-induced IL-12 production in monocytes/macrophages by interfering with JNK activation. J Immunol 190:5142-5151 